\title{
EARLY-ONSET COLORECTAL CANCER: CLINICO-PATHOLOGICAL IMPLICATIONS (EOCRC)
}

\author{
Meda Laura Comandasu, Emel Suliman, Octavia Rusu, C. Savlovschi, S. Oprescu \\ Emergency University Hospital Bucharest
}

\begin{abstract}
Objective. The purpose of this study is to analyse histopathological and clinical characteristics of EOCRC Colorectal cancer was formerly considered as a disease of senescent age; in the last years, it is a noticeable trend of growing incidence among young people (aged between 20 and 45 years). Few of newly diagnosed cases are inherited and most of them are sporadic.

Material and method. The authors studied retrospectively a series of 33 cases of early onset colorectal cancer, 17 men and 16 women, with ages below 45 years, admitted between January 2009 and January 2015 in II and IV Surgical Wards of Emergency University Hospital Bucharest.

Results. Colorectal cancer in young adults tends to be an aggressive disease with dominant distal location (68.5\% of all cases), mostly adenocarcinomas (96.6\%) with moderate to poorly differentiated types (51.4\% G2 and G3), diagnosed in advanced stages (57.6\% stages III and IV), with high frequency of complications (33\% presented with peritoneal carcinomatosis and $9 \%$ died during hospitalization).

Conclusions. EOCRC is a heterogenous group regarding etiopathogeny, localization and histopathological features of the tumor, with aggresive histopathological types, diagnosed in advanced stages.

It may be necessary to elaborate new screening protocols for colorectal cancer in young adults and to find clinical and biological markers that are indicating high-risk patients.
\end{abstract}

Keywords: early onset colorectal cancer, colonoscopy, family X-type syndrome, sporadic type colorectal cancer

\section{INTRODUCTION}

Colorectal cancer (CRC) represents the most frequent cause of neoplasia in Europe (1) and the third cause of death by cancer in the world. In USA, 150,000 new cases are diagnosed per year and 50,000 death occurs in the same period of time. In Europe, the incidence is of 30-50 new cases per year in 100,000 inhabitants. (2-4)

CRC was formerly considered as a disease of senescent age, with average onset at 70 years; more than $90 \%$ of the cases are diagnosed in patients over 55 years of age. Although the number of newly diagnosed cases has a general tendency of decrease, the number of CRC cases among young patients (aged between 20 and 49 years) is increasingly high. EOCRC represents $2-8 \%$ of the total amount of all CRC cases and it's incidence is growing by $1.5 \%$ per year in USA (5). EOCRC displays some characteristics: it localizes mainly in the left colon (especially rectum) and it has aggressive, poorly differentiated cellularity.

CRC in young patients may be inherited or acquired. Hereditary CRC is determined by familial adenomatous polyposis (FAP) which accounts for $1-2 \%$ of the total number of CRC and by hereditary nonpolyposic colorectal cancer (HNPCC), formerly known as Lynch syndrome, which determines $2-4 \%$ of the total amount of CRC and up to one third of EOCRC. (1) HNPCC is inherited in a dominant autosomal manner and it's associated with a risk of almost $80 \%$ of developping CRC/ other cancers at a mean age of 45 years $(6,7)$. Mollecular base of HNPCC is represented by a defect in mismatch repair genes, which determines a subsequent instability of microsatellites (MSI). The colonic tumors in HNPCC are localized mainly in the right colon, with poorly differentiated cellula-

Corresponding author:

Meda Comandasu, MD, „Carol Davila“ University of Medicine and Pharmacy, Bucharest, Romania; IV Surgical Ward, Emergency University Hospital Bucharest

E-mail: meda.comandasu@gmail.com 
rity, with a higher mucin production and are offten multiple (synchronous or metachronous).

Acquired types of CRC are determined by chronic inflammatory bowel diseases (up to 1\%). Most CRC and EOCRC are sporadic-type.

\section{MATERIAL AND METHOD}

The present survey was conducted retrospectively and it's main goal was to asses the characteristics of EOCRC.

The authors have analized 648 patients diagnosed with CRC in II and IV Surgical Wards in Emergency University Hospital Bucharest, between January 2009 and January 2015; 33 of them (5.5\%) were young men and women, aged below 45 years.

Inclusion criteria was the diagnosis of CRC in young patients (below the age of 45 years). None of the patients had FAP, neither chronic infflamatory bowel diseases. One patient had HNPCC; the others presented sporadic-type CRC characteristics. Two patients were excluded because they did not have the results of the histopathological exam.

\section{RESULTS}

The survey includes 33 patients, distributed in the following manner: one patient below 25 years, two patients between 25 and 30 years, 11 patients between 30 and 35 years, three patients between 35 and 40 years, 16 patients between 40 and 45 years. Most patients (48.5\%) were included into the 40-45 years age group.

Mean age of onset was 37.5 years, with a minimum age of 18 years and a maximum age of enrollment of 45 years.

Sex ratio was approximately one; the series includes 17 men (51.5\%) and 16 women (48.5\%).

Similarly, the number of patients who originated in rural areas (54.6\%) or urban areas (45.4\%) was quite similar.

Main symptom was rectal bleeding that occurred in 17 patients (51.5\%), followed by subacute bowel obstruction syndrome in 10 cases (30.3\%). Abdominal pain was described in five cases (15.1\%). Signs and symptoms are represented in Table 1.

A percent of $15.5 \%$ of the patients presented with acute onset (bowel obstruction in three cases, gastrointestinal bleeding-one case, non-cardiac syncope-one case). In other 30 patients (84.9\%), the symptoms evolved gradually.

Thirty-three patients had a number of 35 tumors; $18(51.4 \%)$ were located in rectum, six of them (17.1\%) originated in sigmoid colon. Localization of neoplasias is figured in Table 2.

TABLE 1. Onset signs and symptoms
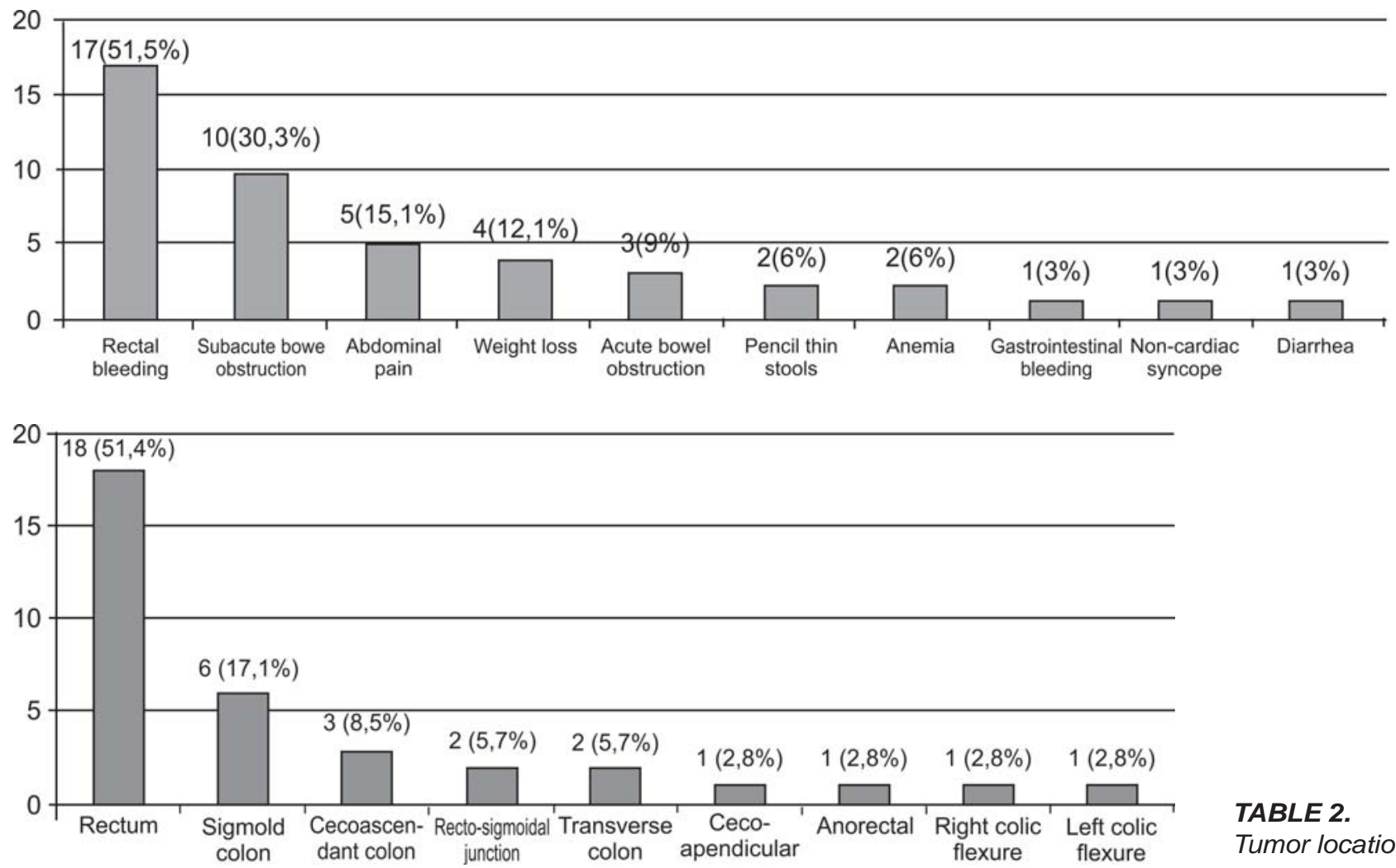

TABLE 2.

Tumor location 
There were two patients with synchronous CRC in rectum and cecum (with „signet ring“ cell) and in ascendant colon and cecum (with good cellular differentiation, but with regional extent), see Figures 1 and 2. Both were diagnosed with peritoneal carcinomatosis. One patient (woman, 42 years) presented with non-keratinized squamos cell invasive ano-rectal carcinoma, with poor cellular differentiation and regional extent. After rectal abdomino-perineal resection, she was submitted to postoperative radiotherapy.

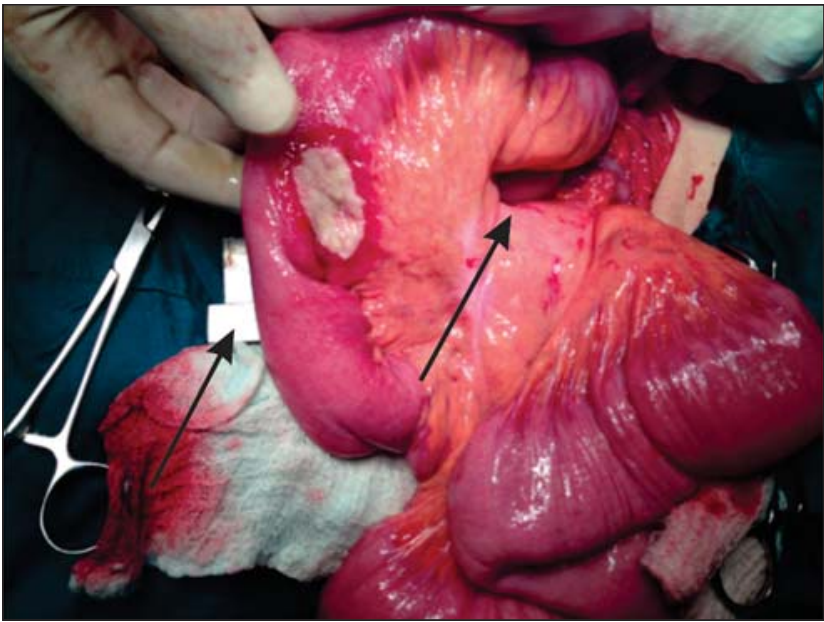

FIGURE 1. Surgical specimen - synchronous colorectal cancers (perforated cecal tumor, stenotic ascending colon tumor)

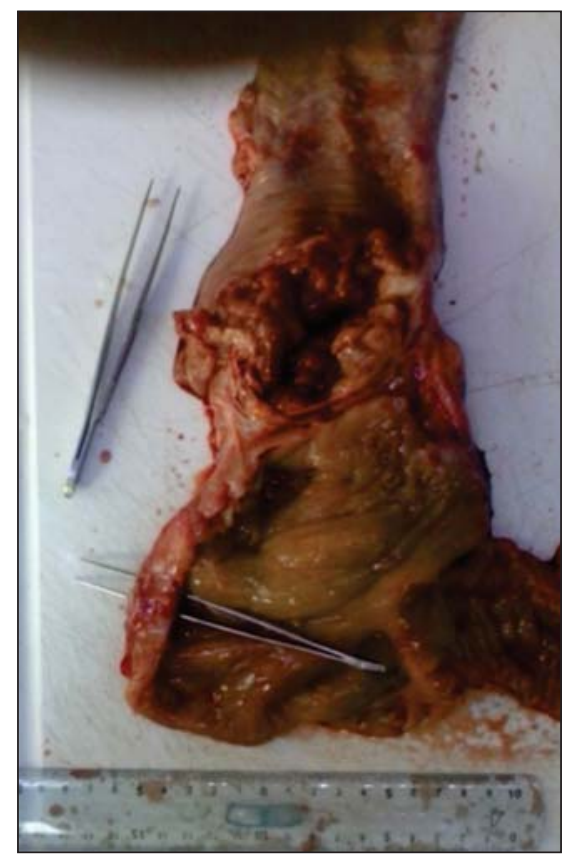

FIGURE 2. Same surgical specimen, after transsection

As concerned histopathological findings, in 32 patients resected tumors were adenocarcinomas
(96.6\%), with variable degrees of differentiation. In 16 patients (48.4\%), cell differentiation was good (G1 grade tumor), in 8 cases (24.2\%) the differentiation was moderate (G2) and 9 patients (27.2\%) had tumor staged G3. Nineteen patients (57.6\%) were diagnosed in stage 111 and $\mathrm{lV}$, e.g. in advanced stages.

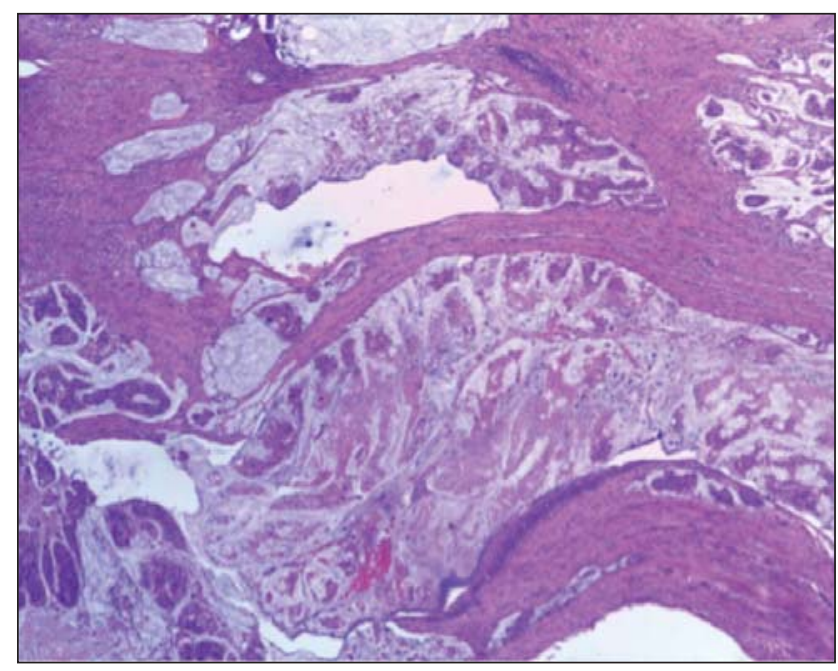

FIGURE 3. HE staining 4x. Mucinous adenocarcinoma: glandular structures among isolated, mucin pools that are invading glandular cells

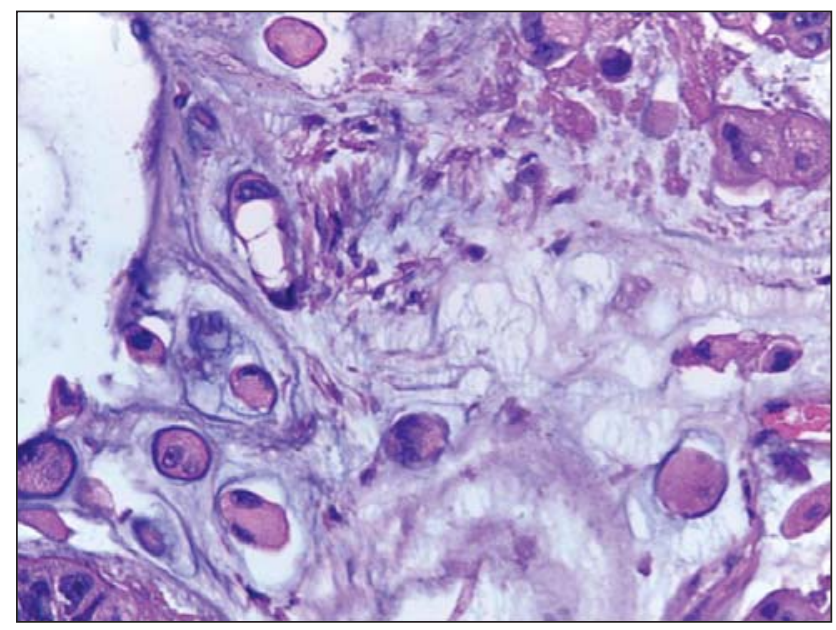

FIGURE 4. HE staining 40x. "Signet ring" cell adenocarcinoma: mucin pool with atypical glandular cells; some of them are „signet ring“ type; detail image

Five cases (15\%) presented with mucinous tumors expressing vascular and perineural invasion (Figure 3) and one case (3\%) with ,signet ring“ cell type (Figure 4).

Thirty-two out of 33 patients were operated. One case was directed to palliative radiotherapy due to the advanced stage of the disease. 
In 16 cases, patients were submitted to oncological radical intent surgery, as followed:

- eight cases with anterior rectal resection; in four cases, digestive continuity was reestablished by colo-rectal anastomosis and in the other four, by pull-through procedure with colo-anal anastomosis;

- five cases with abdomino-perineal resection;

- three cases with right hemi-colectomy with ileo-colic anastomosis.

For the rest of patients, the surgical attitude included palliative interventions and resections by necessity:

- eight cases of segmental resection of the sigmoid colon;

- six cases of Hartmann's procedure;

- two cases of exploratory laparotomy;

- in two female patients the colic surgery was associated with total hysterectomy and salpingectomy.

Eleven patients (33\%) presented with complicated disease e.g. peritoneal carcinomatosis and three of them died during hospitalization period ( $9 \%$ of all cases).

Surprisingly, in this case series, only one patient fullfils Amsterdam II criteria reffering to HNPCC (onset below 45 years, three members of the family having a diagnosed CRC, one beeing first degree relative of the other two and two generations affected); the remaining patients are fulfilling Bethesda modified guidelines for microsatellite instability. Allthough it would have been recommendable, patients did not had the financial possibility for immunohistochemical testing. Even if IHC/MSI testing are not available, the pathologist can raise suspicion of HNPCC and MSI by evaluating tumor infiltrating lymphocytes (8). All surviving patients were referred to Oncology Clinic for chemotherapy.

\section{DISCUSSIONS}

The study follows 33 patients diagnosed between 2009 and 2015 with early onset CRC (aged from 18 to 45 years), which represents 5.5\% of all cases of CRC admitted in our clinics in this period of time.

The „early onset“ concept is not well defined in literature, the maximum „young age“ varies in different studies from 40 to 50 years $(1,5,9,10)$. The authors established 45 years as maximum enrollment age because the incidence of newly diagnosed cases rises markedly after $\mathbf{4 5}$ years. In this series, most patients were found in the 40-45 age group.

There is no significant differences in male-female ratio, neither in patients from rural or urban areas.

Five patients (15.5\%) had emergent presentation, with acute bowel obstruction, acute gastrointestinal bleeding and non-cardiac syncope. In the rest of the cases, symptomatology progressed gradually, with minimal rectal bleeding in 17 patients (51.5\%), altered bowel habits e.g. constipation in 30,3\% and abdominal pain (15.1\%).

Rectum was the dominant site of tumor location in 18 patients $(51.4 \%)$.

Two patients presented with two synchronous CRC, both discovered intraoperatively, each with two concomittant tumors, in cecum and rectum (in one case) and in cecum and ascending colon in the other. It was also a patient with squamos cell nonkeratinized ano-rectal carcinoma.

Tumors were adenocarcinomas in $96.6 \%$ of cases; 19 patients (57.6\%) were diagnosed in advanced stages of disease (III and IV). It predominated distal location of neoplasia (68.5\%). (11)

Sixteen of 32 patients underwent curative resection: anterior rectal resection in eight cases, abdomino-perineal resection in five cases, right hemicolectomy in three cases. Anal sphincter function is important especially because of patient's young age and must be preserved whenever it is possible.

Fourteen patients (43.7\%) underwent palliative procedures such as Hartmann's procedure (six operations) and colectomy with colo-colic anastomosis (eight operations).

Exploratory laparotomy was performed in two situations, due to the advanced stage of the disease (peritoneal carcinomatosis with ascites and hepatic metastases). Both patients died in the early postoperative period.

Intrahospital deaths occured in a total of three cases (9\%), which represents a high percent of the series and it is determined by the advanced stage of the disease (57.6\% of the patients diagnosed in stage III and IV of the disease) the aggresivenes of histological types (51.5\% tumors with medium and poor cell differentiation), delayed diagnosis and acute complications that imposed emergent surgery.

The patients displayed a small number of colorectal adenomas that were associated with CRC (only three cases); this may suggest accceler- 
ated carcinogenesis due to predisposing conditions. (1)

Allthough there was only one patient with typical features for HNPCC (Amsterdam II criteria), other three had autosomal transmitted digestive neoplasms, fact that suggests the presence of „X syndrome“. Family CRC X syndrome is a type of hereditary, non-polyposic cancer, localized on the left colon and in rectum, with early onset and yet unknown mollecular basis $(12,13)$.

Frequency of complications was high: $33 \%$ of the patients presented with peritoneal carcinomatosis and 9\% died in the time of hospitalization.

\section{CONCLUSIONS}

1. EOCRC is a heterogenous group regarding etiopathogeny, localization and histopathological features of the tumor.

2. EOCRC represents 2-8\% of all CRC in literature and 5,5\% in our series $(14,15)$.

3. It presents mainly as a sporadic form (32 of our 33 enrolled patients) and it is seldom generated by HNPCC or other predispozing conditions.

4. EOCRC localizes especially in the left colon (67.5\% in our series).

5. It is frequently diagnosed in advanced stages of the disease, often metastasized.
6. Histopathological findings are often displaying agressive forms (mucinous adenocarcinoma, „signet ring“ cell adenocarcinoma) $(16,17)$.

7. Efficacy of screening programmes after the age of 50 years is well documented, but they are none for adolescent and young adult at medium risk for CRC (without personal or familial history of adenoma/CRC, without inflammatory bowel disease) NCCN Guidelines. (18)

8. Thereby, it appears necessary to elaborate screening protocols and monitoring programmes for young people, fitting their needs.

9. Multidisciplinary teams should asses the mechanisms of carcinogenesis and identify new markers suited for EOCRC.

10. It may exist a new hereditary syndrome, with yet unknown molecular and genetic basis e.g. family X-type CRC-which necessitates and imposes some deepen studies.

\section{ACKNOWLEDGEMENT}

This work received financial support through the project entitled „CERO - Career profile: Romanian Researcher“, grant number POSDRU/159/ 1.5/S/135760, co-financed by the European Social Fund for Sectorial Operational Program Human Resources Development 2007-2013.

\section{REFERENCES}

1. Kirzin S., Marisa L., Guimbaud R., et al. Sporadic Early-Onset Colorectal Cancer Is a Specific Sub-Type of Cancer: A Morphological, Molecular and Genetics Study. Cheah PY, ed. PLOS ONE. 2014;9(8):e103159. doi:10.1371/journal.pone.0103159

2. Sandouk F., Jerf F.A., Bassel Al-Halabi M.H.D., „Precancerous Lesions in Colorectal Cancer", Gastroenterology Research and Practice, vol. 2013, Article ID 457901, 11 pages, 2013. doi:10.1155/2013/457901

3. Perea J., Rueda D., Canal A., et al. Age at onset should be a major criterion for subclassification of colorectal cancer. J Mol Diagn. 2014;16:116-126

4. Stoffel E.M., Mangu P.B., Gruber S.B., et al., Hereditary Colorectal Cancer Syndromes: American Society of Clinical Oncology Clinical Practice Guideline Endorsement of the Familial Risk - Colorectal Cancer: European Society for Medical Oncology Clinical Practice Guidelines, American Society of Clinical Oncology, 2014

5. Siegel R.L., Jemal A., Ward E.M., Increase in Incidence of Colorectal Cancer Among Young Men and Women in the United States Cancer Epidemiol Biomarkers Prev June 2009 18:1695-1698; doi:10.1158/1055-9965.EPI-09-0186
6. Guttmacher A.E., Collins F.S., Hereditary Colorectal Cancer N Engl J Med 2003; 348:919-932March 6, 2003DOI: 10.1056/NEJMra012242

7. Jasperson K.W., Vu T.M., Schwab A.L., et al. (2010). Evaluating Lynch syndrome in very early onset colorectal cancer probands without apparent polyposis. Familial Cancer, 9(2), 99-107. doi:10.1007/s10689-009-9290-4

8. Umar A., Boland C.R., Terdiman J.P., et al. (2004). Revised Bethesda Guidelines for Hereditary Nonpolyposis Colorectal Cancer (Lynch Syndrome) and Microsatellite Instability. Journal of the National Cancer Institute, 96(4), 261-268

9. Meyer J.E., Narang T., Schnoll-Sussman F.H., et al. (2010). Increasing Incidence of Rectal Cancer in Patients Aged Younger Than 40 Years: An Analysis of the Surveillance, Epidemiology, and End Results Database. Cancer, 116(18), 4354-4359. doi:10.1002/ cncr.25432

10. Fairley T.L., Cardinez C.J., Martin J., et al. Colorectal cancer in U.S. adults younger than 50 years of age, 1998-2001. Cancer. 2006 Sep 1;107(5 Suppl):1153-61.

11. Fleming M., Ravula S., Tatishchev et al. (2012). Colorectal carcinoma: Pathologic aspects. Journal of Gastrointestinal Oncology, 3(3), 153-173. doi:10.3978/j.issn.2078-6891.2012.030 
12. Stigliano V., Sanchez-Mete L., Martayan A. et al. (2014). Earlyonset colorectal cancer: A sporadic or inherited disease? World Journal of Gastroenterology : WJG, 20(35), 12420-12430. doi:10.3748/wjg.v20.i35.12420

13. Valle L. (2014). Genetic predisposition to colorectal cancer: Where we stand and future perspectives. World Journal of Gastroenterology WJG, 20(29), 9828-9849. doi:10.3748/wjg.v20.i29.9828

14. European Colorectal Cancer Screening Guidelines Working Group, von Karsa L., Patnick J., Segnan N., et al European guidelines for quality assurance in colorectal cancer screening and diagnosis: overview and introduction to the full supplement publication. Endoscopy. 2013;45:51-59

15. Perea J., Alvaro E., Rodríguez Y. et al. Approach to early-onset colorectal cancer: clinicopathological, familial, molecular and immunohistochemical characteristics. World J Gastroenterol. 2010;16:3697-3703

16. Stigliano V., Sanchez-Mete L., Martayan A. et al. (2014). Earlyonset colorectal cancer patients without family history are „at very low risk" for lynch syndrome. Journal of Experimental \& Clinical Cancer Research : CR, 33(1), 1. doi:10.1186/1756-9966-33-1

17. You Y.N., Dozois E.J., Boardman L.A. et al. Young-onset rectal cancer: presentation, pattern of care and long-term oncologic outcomes compared to a matched older-onset cohort. Ann Surg Oncol. 2011;18:2469-2476

18. NCCN Clinical Practice Guidelines in Oncology: 2014 